\title{
Limitations of Maxwell's distribution
}

\author{
A calculation of the evolution of a system of gravitating masses (galaxies) in an expanding \\ universe surprisingly suggests that Maxwell's velocity distribution is not an acceptable end point.
}

EVERYBODY knows that if you put a collection of massive interacting particles in a box and let them settle down, in due course their velocities will have a maxwellian distribution. The interaction may be only sporadic, physical collision, as in a classical monatomic gas for example, or otherwise be everpresent, as with the long-range forces of gravitation. Either way, the end result is that the chance that the velocity of a particle lies between $v$ and $v+\mathrm{d} v$ is proportional to

$$
v^{2} \exp \left(-1 / 2 m v^{2} / \mathrm{k} T\right) \mathrm{d} v
$$

where $m$ is the particle mass, $T$ the temperature and $\mathrm{k}$ is Boltzmann's constant. For what it is worth, the proportionality constant is $4 \pi(m / 2 \pi \mathrm{k} T)^{3 / 2}$. Maxwell's first flawed derivation of this distribution function in $\mathbf{1 8 6 0}$ was afterwards recast along the lines of Ehrenfest's much later principle of detailed balancing at equilibrium, the rate at which particles are removed from some velocity interval will be equal to the rate at which particles with such velocities are produced, by collision or otherwise. By 1878 , Boltzmann had put kinetic theory on an analytical basis with his integro-differential equation admitting only permissible distribution functions as solutions. During the First World War, Enskog and then Chapman showed how the maxwellian distribution is the end point in the evolution in time of an arbitrary initial velocity distribution, at least if interactions between the particles dissipate no kinetic energy. So what happens if the interacting massive particles are not atoms of a gas but a collection of galaxies (for simplicity, with equal mass) and if the box into which they are put is the expanding universe?

Certainly not a maxwellian distribution, as can be told from the treatment of this problem by Henry E. Kandrup of the University of California, Santa Barbara, published in an August issue of Astrophysical Journal $(259,1 ; 1982)$. The problem is of course relevant to the current preoccupation in cosmology with the formation of clusters of galaxies. While many hold to the theory of Zel'dovich and his colleagues that galaxy clusters are formed by the internal coalescence of large pancake-shaped aggregations of matter, recent Monte Carlo simulations of the evolution of gravitating masses suggest that clustering may be a natural outcome of a different starting point - a collection of galaxysized objects in an expanding box. Kandrup's study is an attempt at an analytical treatment of the problem hitherto dealt with primarily by numerical modelling. The most striking of his conclusions is that if you put a collection of identical massive objects (galaxies) in an expanding box (universe) and take proper account of their long-range interaction through gravitation, then you find that the total kinetic energy of the massive objects increases monotonically with time. Naturally, the steady increase of kinetic energy must be compensated for somehow - by what Kandrup calls the "generation of negative correlational energy", presumably another name for potential energy. In short, the calculation suggests that clustering should be a natural occurrence, while the steady increase of kinetic energy is a direct proof that a maxwellian distribution is entirely out of court.

On a little reflection, this conclusion should not be as surprising as it seems. For the expanding universe is hardly a system in equilibrium, and since Enskog's inaugural dissertation at the
University of Uppsala in 1917, it has been in principle possible to calculate deviations from simple maxwellian distributions in the many familiar physical circumstances in which equilibrium does not obtain, the transport of heat through a kinetic gas for example, or the transport of momentum as in viscous phenomena. Kandrup acknowledges the way in which Prigogine and his associates have more recently dealt with non-equilibrium systems, showing how circumstances can arise in which a system can be deflected from relaxation towards a maxwellian distribution. With all this said, however, the physical implications of these phenomena are not at all well understood. Is the time ripe for revival of interest in classical kinetic theory?

Kandrup's analysis of his own problem of what happens to a collection of gravitating point masses in an expanding universe contains a further and perhaps deeper surprise. The calculation starts from a time-dependent distribution function for the values of each of the independent variables (space coordinates and velocities) for each of the galactic masses, whereupon it is possible to relate the time-rate of change of the distribution function to the space gradient of the distribution function and to the forces between particles. The procedure is strictly analogous to the direction of Liousville's theorem, and says little more than that probability is conserved. The algebra, however, is complicated by the need to work in an appropriate and moving reference frame. Exactly by analogy with classical kinetic theory, Kandrup nevertheless sets out to calculate a one-particle distribution function by integrating over the coordinates of all other masses and arrives at the equivalent of Boltzmann's equation for the time-rate of change of that same function. Analytical solutions of this equation are for the time being out of reach, if only because of the complications flowing from the $1 / r$ form of the gravitational potential, but there are approximations that put the analogue of Boltzmann's equation into barely manageable form. And Kandrup's conclusion is that in these circumstances, a maxwellian distribution of velocities is not a stationary solution of the equation. And, Kandrup insists, this unexpected result "has nothing to do with the fact that the universe is expanding"' but is directly a consequence of the long-range character of the gravitational forces between all the particles.

The implications of Kandrup's calculation for the understanding of the clustering of galaxies remain to be seen. The fact that the monotonic increase of kinetic energy which tumbles out of the calculations implies a decrease of potential energy which is at least consistent with the formation of galaxy clusters is of at least more than passing interest. But Kandrup's approximations deserve to be scrutinized with great care by those with an interest in his problem of galaxies in an expanding box as a test of simple classical kinetic theory. Hitherto, attempts to calculate velocity distribution functions in circumstances where particles interact by means of inverse-square law forces - in ionized gases, for example - have tended to be frustrated by the recognition that 'collisions' are not simple binary collisions when such longrange forces are involved. If Kandrup has found a way round this kind of difficulty, he will no doubt be embraced not merely by cosmologists but by plasma physicists as well. 